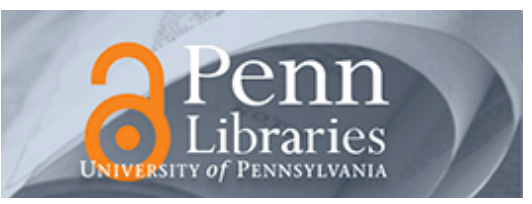

University of Pennsylvania ScholarlyCommons

January 2004

\title{
Small angle neutron scattering from single-wall carbon nanotube suspensions: evidence for isolated rigid rods and rod networks
}

Wei Zhou

University of Pennsylvania

M. F. Islam

University of Pennsylvania

H. Wang

Michigan Technological University

D. L. Ho

National Institute of Standards and Technology

A. G. Yodh

University of Pennsylvania

See next page for additional authors

Follow this and additional works at: https://repository.upenn.edu/mse_papers

\section{Recommended Citation}

Zhou, W., Islam, M. F., Wang, H., Ho, D. L., Yodh, A. G., Winey, K. I., \& Fischer, J. E. (2004). Small angle neutron scattering from single-wall carbon nanotube suspensions: evidence for isolated rigid rods and rod networks. Retrieved from https://repository.upenn.edu/mse_papers/61

Postprint version. Published in Chemical Physics Letters, Volume 384, Issues 1-3, 19 January 2004, pages 185-189.

Publisher URL: http://dx.doi.org/10.1016/j.cplett.2003.11.106

This paper is posted at ScholarlyCommons. https://repository.upenn.edu/mse_papers/61

For more information, please contact repository@pobox.upenn.edu. 


\title{
Small angle neutron scattering from single-wall carbon nanotube suspensions: evidence for isolated rigid rods and rod networks
}

\author{
Abstract \\ We report small angle neutron scattering (SANS) from dilute suspensions of purified individual single wall \\ carbon nanotubes (SWNTs) in $\mathrm{D}_{2} \mathrm{O}$ with added sodium dodecylbenzene sulfonate (NaDDBS) ionic \\ surfactant. The scattered intensity scales as $Q^{-1}$ for scattered wave vector, $Q$, in the range $0.005<Q<$ \\ $0.02 \AA^{-1}$. The $Q^{-1}$ behavior is characteristic of isolated rigid rods. A crossover of the scattered intensity \\ power law dependence from $Q^{-1}$ to $Q^{-2}$ is observed at $\sim 0.004 \AA^{-1}$, suggesting the SWNTs form a loose \\ network at $0.1 \mathrm{wt} \%$ with a mesh size of $\sim 160 \mathrm{~nm}$. SANS profiles from several other dispersions of SWNTs \\ do not exhibit isolated rigid rod behavior; evidently the SWNTs in these systems are not isolated and form \\ aggregates.
}

\section{Comments}

Postprint version. Published in Chemical Physics Letters, Volume 384, Issues 1-3, 19 January 2004, pages 185-189.

Publisher URL: http://dx.doi.org/10.1016/j.cplett.2003.11.106

\section{Author(s)}

Wei Zhou, M. F. Islam, H. Wang, D. L. Ho, A. G. Yodh, Karen I. Winey, and John E. Fischer 


\section{Small angle neutron scattering from single-wall carbon}

nanotube suspensions: evidence for isolated rigid rods and rod

\section{networks}

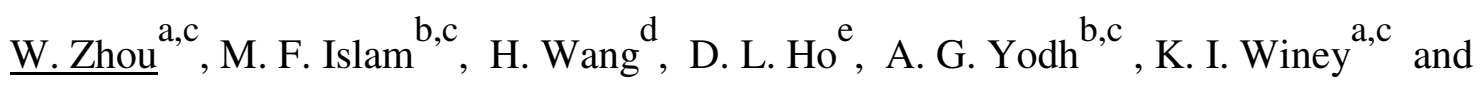
J. E. Fischer ${ }^{\mathrm{a}, \mathrm{c}, *}$

${ }^{\mathrm{a}}$ Department of Materials Science and Engineering ${ }^{b}$ Department of Physics and Astronomy

${ }^{c}$ Laboratory for Research on the Structure of Matter University of Pennsylvania, Philadelphia PA 19104

${ }^{\mathrm{d}}$ Materials Science and Engineering Department

Michigan Technological University, Houghton, MI 49931

${ }^{e}$ NIST Center for Neutron Research

National Institute of Standards and Technology, Gaithersburg, MD 20899

*e-mail: fischer@lrsm.upenn.edu 
We report small angle neutron scattering (SANS) from dilute suspensions of purified individual single wall carbon nanotubes (SWNTs) in $\mathrm{D}_{2} \mathrm{O}$ with added sodium dodecylbenzene sulfonate (NaDDBS) ionic surfactant. The scattered intensity scales as $Q^{-1}$ for scattered wave vector, $Q$, in the range $0.005<Q<0.02 \AA^{-1}$. The $Q^{-1}$ behavior is characteristic of isolated rigid rods. A crossover of the scattered intensity power law dependence from $Q^{-1}$ to $Q^{-2}$ is observed at $\sim 0.004 \AA^{-1}$, suggesting the SWNTs form a loose network at $0.1 \mathrm{wt} \%$ with a mesh size of $\sim 160 \mathrm{~nm}$. SANS profiles from several other dispersions of SWNTs do not exhibit isolated rigid rod behavior; evidently the SWNTs in these systems are not isolated and form aggregates. 
Isolated single-wall carbon nanotubes (SWNTs) are one-dimensional objects with extraordinary mechanical, electrical, optical and thermal properties [1]. Individual SWNTs have diameters of $1-2 \mathrm{~nm}$ and lengths ranging from $\sim 100 \mathrm{~nm}$ to several $\mu \mathrm{m}$. The morphology of nanotubes in suspension has not been well characterized, and an improved understanding of suspension structure should in turn lead to better control of solution phase separation, chemical derivatization, and optimization of the physical properties of SWNT composites.

Small angle x-ray, neutron and light scattering techniques for wave vectors $Q$ in the range $10^{-4}-10^{-1} \AA^{-1}$ (corresponding to length scales 1-1000 nm) are ideal for investigating the structure of SWNTs in suspension. For example, the scattered intensity, $I$, from a suspension of isolated rigid rods with diameter $D$ and length $L$ follows a $Q^{-1}$ law for wave vectors $2 \pi / L<Q<2 \pi / D$ [2]. Several groups have used scattering methods to study the structure of single-wall and multi-wall nanotubes (MWNTs) in suspensions and in polymer composites. For MWNTs the scattered intensity exhibits a $Q^{-1}$ dependence in suspensions and in polymer composites, suggesting the presence of isolated rigid rods in the sample [3, 4, E. K. Hobbie, NIST/NASA Purity and Dispersion Measurement Issues Workshop on Single-Wall Carbon Nanotubes, NIST, May 27-29, 2003]. On the other hand, recent measurements of SWNTs in suspension and in polymer composites exhibit power law dependences with exponents in the range -2 to -3 ; the authors of these papers suggest the configurations of nanotubes in such suspensions and composites are random coil or non-rigid rod-like structures [4, E. K. Hobbie, NIST/NASA Purity and Dispersion Measurement Issues Workshop on Single-Wall Carbon Nanotubes, NIST, May 27-29, 2003]. This was perhaps somewhat surprising since theoretical predictions and 
mechanical measurements of the modulus of SWNTs $[5,6]$ indicate very large persistence lengths $(\sim 50 \mathrm{~mm})$ [7], and therefore, individual SWNTs a few microns long (and less) should behave as rigid rods.

In this paper we report a small angle neutron scattering (SANS) study of SWNT suspensions in $\mathrm{D}_{2} \mathrm{O}$. The investigation provides strong evidence for individual SWNTs and very small bundles in suspension which behave like rigid rods. We also observe a crossover in the power law $Q$-dependence of scattered intensity $I$ from -1 to approximately -2 at low $Q$, suggesting that these isolated tubes and small bundles may form a loose three-dimensional network. On the other hand, in aggregating suspensions we found that agglomerates of SWNTs do not exhibit the scattering behavior characteristic of isolated rigid rods.

Dispersions of purified HiPco SWNTs (high pressure catalytic decomposition of CO) with < 1 wt\% Fe [8,9] (batch 79), and Tubes@Rice SWNTs (pulsed laser vaporization) with $<5 \mathrm{wt} \% \mathrm{Ni}$ and $\mathrm{Co}[10]$ were prepared in $\mathrm{D}_{2} \mathrm{O}$ using sodium dodecylbenzene sulfonate (NaDDBS) according to the previously published procedure [11]. Briefly, nanotube/surfactant mixtures were sonicated for $24 \mathrm{~h}$ in a low-power, high frequency sonicator, maintaining a SWNT/NaDDBS mass ratio of 1:10 for all SWNT/D $2 \mathrm{O}$ concentrations. They were directly loaded into quartz banjo cells for SANS measurement without centrifugation or filtration, in order to maintain the initial concentrations. These suspensions were visually homogeneous and remained stable for months; they were not turbid. Atomic force microscope (AFM) height/length histograms show that HiPce NaDDBS and Tubes@Rice-NaDDBS dispersions at a concentration of $0.01 \mathrm{wt} \%$ were $74 \pm 5 \%$ single tubes with mean length of $165 \pm 95 \mathrm{~nm}$ and $90 \pm 5 \%$ 
single tubes with mean length of $516 \pm 286 \mathrm{~nm}$, respectively [11]. It is reasonable to assume that the adsorption of surfactant molecules on SWNTs surfaces (the surfactant layer is $\sim 2 \mathrm{~nm}$ thick) does not alter the one-dimensional characteristics of SWNTs. Furthermore, nanotube dispersions with concentrations greater than 0.01 wt\% SWNTs, and thereby $0.1 \mathrm{wt} \%$ NaDDBS, likely contain spherical micelles of free surfactant in addition to the SWNT-NaDDBS complexes (the critical micelle concentration, CMC, for NaDDBS in water is $\sim 0.048 \mathrm{wt} \%)$.

We also prepared a few other suspensions of SWNTs for study: HiPco in $\mathrm{D}_{2} \mathrm{O}$ using Triton X-100, HiPco in deuterated toluene, and HiPco dispersed in a poly(methyl methacrylate) (PMMA) matrix [12]. The HiPco-Triton X-100 suspension in $\mathrm{D}_{2} \mathrm{O}$ had $\sim 36 \%$ single tubes at $0.01 \mathrm{wt} \%$ SWNTs (AFM) without filtration or centrifugation [11]. HiPco tubes also formed aggregates in deuterated toluene and in PMMA. Surfactant solutions in $\mathrm{D}_{2} \mathrm{O}$ without nanotubes were prepared as controls; these too were used directly and exhibited no turbidity after several months.

SANS experiments were carried out at room temperature using the $30-\mathrm{m}$ instrument at the National Institute of Standards and Technology (NIST) Center for Neutron Research (NCNR). The scattered intensity was corrected for background (e.g., stray neutrons, incoherent proton scattering, quartz cell etc.). An absolute $I$ scale was established using a calibrated secondary standard. A large $Q$ range of $0.0008 \AA^{-1}$ to 0.7 $\AA^{-1}$ was obtained by using three different instrumental configurations.

Scattering profiles $I(Q)$ for the surfactant $\mathrm{NaDDBS}$ in $\mathrm{D}_{2} \mathrm{O}$ with concentrations $c_{\text {NaDDBS }}$ varying from $0.05 \mathrm{wt} \%$ to $5 \mathrm{wt} \%$ are shown in Fig. 1(a). The scattered intensity $I(Q)$ exhibits a broad correlation peak at around $Q=0.07 \AA^{-1}$ for $0.5,1$ and $5 \mathrm{wt} \%$ due to 
the existence of spherical micelles. The peak sharpens and shifts to higher $Q$ with increasing concentration as a result of increased interactions between micelles [13]. For $c_{\mathrm{NaDDBS}}=0.05 \mathrm{wt} \%$, there is no visible correlation peak, presumably because the surfactant concentration is close to the CMC and there are few (if any) micelles in solution. We also noticed a dramatic increase in $I(Q)$ with decreasing $Q$ in all samples, starting between $0.003-0.005 \AA^{-1}$. We do not understand the origin of this behavior, which is unusual for surfactants.

The SANS intensity profiles from HiPco-NaDDBS and Tubes@Rice-NaDDBS suspensions in $\mathrm{D}_{2} \mathrm{O}$ with nanotube concentrations, $c_{\mathrm{SWNTs}}=0.01,0.05$ and $0.1 \mathrm{wt} \%$ are shown in Fig. 1(b) and 1(c), respectively. The nanotube-to-surfactant ratio was 1:10 for all SWNTs suspensions. Both the $0.1 \mathrm{wt} \%$ and $0.05 \mathrm{wt} \%$ sample exhibit $Q^{-1}$ behavior over limited $Q$ ranges, while the effect is less pronounced at the lowest concentration of tubes. The excess surfactant contribution is clearly evident from the correlation peak and the diverging intensity as $Q \rightarrow 0$.

To study the structure of SWNTs in suspension, we must derive the scattered neutron intensity due to the SWNT-NaDDBS complexes, $I_{\text {SWNT-NaDDBS. Since the }}$ SWNT-NaDDBS suspension contains both SWNT-NaDDBS complexes and micelles formed by free surfactant, we subtract the contribution of the micelles, $I_{\text {micelles}}$, from the total intensity, $I_{\text {total }}$ (i.e., intensities given in Fig. 1(b) and 1(c)). To this end, we studied the scattered intensity from solutions with fixed concentration of NaDDBS (1 wt $\%)$, but with differing SWNT concentrations of $0,0.01,0.05$ and $0.1 \mathrm{wt} \%$. Our hypothesis was that, beginning with a low concentration, as surfactant molecules adsorb onto additional 
SWNTs the number of micelles will decrease and the intensity of the micelle correlation peak at $Q=0.07 \AA^{-1}$ will decrease accordingly. SANS profiles from these suspensions are shown in Fig. 1(d). The micelle correlation peak at $Q=0.07 \AA^{-1}$ is only weakly affected by the addition of SWNTs, indicating that the majority of surfactant molecules remained free and formed micelles. We also found that the SWNT-NaDDBS complexes dominate $I$ for $Q<0.07 \AA^{-1}$.

Since we could not determine $I_{\text {micelles }}$ directly for the SWNT-NaDDBS suspensions, we subtracted $I_{\text {micelles }}$ for various fractions of excess surfactants from $I_{\text {total }}$. In Fig. 2 we show the results of subtraction for both HiPco and Tubes@ Rice with 0.1 wt $\%$ and $0.05 \mathrm{wt} \%$ SWNT concentrations. Dashed and dotted lines correspond to the extreme assumptions of no excess surfactant and no surfactant on the tubes, respectively. For $0.1 \mathrm{wt} \%$ we observe a $Q^{-1}$ slope in the range $0.003-0.02 \AA^{-1}$, regardless of the fraction of surfactant intensity subtracted. Similarly, for $0.05 \mathrm{wt} \%$ suspensions, a $Q^{-1}$ slope in the range $0.005-0.02 \AA^{-1}$ is observed. We emphasize this subtraction procedure is only reliable in the intermediate $Q$ range. For reliable subtraction at high $Q$, an independent measurement of micelles in the SWNT-NaDDBS suspension is needed. At very low $Q$, the subtraction is more reliable than at high $Q$ because the scattered intensity is dominated by SWNTs-NaDDBS complexes. The resultant observed $Q^{-1}$ behavior over a $Q$ range from 0.003 to $0.02 \AA^{-1}$ is a strong indication that the structure of SWNTs in suspension is that of isolated rigid rods.

The scattered intensities in Fig. 2 also exhibit a crossover from $Q^{-1}$ to $Q^{-2}$ at $\sim 0.004 \AA^{-1}$. This may be connected to tube-tube interactions which lead to the formation of a loose 3-D network, somewhat akin to the semi-dilute phases of flexible polymer 
solutions. The semi-dilute phases of flexible polymers, for example, exhibit a similar $Q^{-2}$ behavior due to their Lorentzian structure factors at low Q; the Lorentzian width is of order the mesh size or correlation length of the network [14]. Although our SWNTs can hardly be thought of as flexible, our suspensions have concentrations 1 to 2 orders of magnitude larger than the overlap concentration $\sim 1 / \mathrm{L}^{3}$ (i.e. $10^{-3} \mathrm{wt} \%$ ), and might more closely resemble semi-dilute suspensions of semi-flexible polymers, particularly as the mesh size approaches the polymer persistence length. We are unaware of any theoretical work predicting the static structure factor of such systems, or for the related system of rigid rod networks. Nevertheless, using the simplified model of a 3-D network of rigid rods, we expect the length scale for the crossover point to be comparable and perhaps slightly smaller than the average rod length. The scattering profiles from SWNTNaDDBS show that the crossover from $Q^{-1}$ to $Q^{-2}$ occurs at $\sim 0.004 \AA^{-1}$ corresponding to a real space length of $\sim 160 \mathrm{~nm}$. This length is indeed comparable to the average tube length [11].

So far, our interpretation of $I(Q)$ from SWNT-NaDDBS suspensions is constrained by the fact that we do not know a priori the concentration of micelles in the nanotube suspensions. To overcome this constraint we performed a contrast matching experiment. Matching between NaDDBS (calculated scattering length density SLD = $0.61 \times 10^{-6} \AA^{-2}$ ) and solvent was achieved by mixing $\mathrm{D}_{2} \mathrm{O}$ with $\mathrm{H}_{2} \mathrm{O}$ in the ratio $1: 4.394$ by weight, leaving the SWNTs (measured SLD $=4.9 \pm 0.1 \times 10^{-6} \AA^{-2}$ by matching D- and Htoluene solvents) as the sole remaining source of contrast with the solvent. This approach drastically reduces the coherent intensity by reducing the total contrast, and the 
incoherent background is greatly increased due to $\mathrm{H}_{2} \mathrm{O}$. The useful region for reliable coherent intensity is thus limited to $Q<0.01 \AA^{-1}$.

The data with error bars from such a $0.02 \mathrm{wt} \%$ nanotube suspension are shown in Fig. 3. After subtracting the incoherent background, the -2 slope at low $Q$ is quite apparent and a distinct change in slope with a fairly convincing -1 slope above $Q=0.003$ $\AA^{-1}$ is observed. These observations reinforce our conclusions drawn from the SWNT$\mathrm{NaDDBS} / \mathrm{D}_{2} \mathrm{O}$ suspensions. A further improvement would be to use deuterated NaDDBS since much less $\mathrm{H}_{2} \mathrm{O}$ would then be required for contrast matching. Unfortunately this scheme would also reduce the nanotube/environment contrast since the contrast between SWNTs and deuterated NaDDBS is small.

Finally, we consider our results from more poorly dispersed SWNT suspensions: HiPco dispersed in $\mathrm{D}_{2} \mathrm{O}$ using Triton $\mathrm{X}-100$, deuterated toluene, and solid PMMA polymer matrix. $I(Q)$ from all of these samples exhibited a power law exponent between -2 and -3 over a broad $Q$ range. In Fig. 4 we show the results for 0.1 wt $\%$ HiPco in $\mathrm{D}_{2} \mathrm{O}$ with $1 \mathrm{wt} \%$ Triton X-100. After intensity subtraction similar to that performed in Figure 2, we obtained a $Q^{-2}$ slope over the entire $Q$ range measured. Clearly the dominant structures in this suspension are not isolated rigid rods. This is consistent with the AFM result which shows Triton X-100 is far less efficient than NaDDBS in terms of dispersing nanotubes [11]. Since isolated tubes, bundles and a large fraction of aggregates coexist in the suspension, and all contribute to the scattered intensity in different ways, it is hard to interpret the $Q^{-2}$ slope based on any simple model. It is quite possible that the aggregating nanotubes form ropes in suspension, and that at sufficiently high 
concentration a 3-D mesh network of ropes is formed which gives rise to the power law behaviour over the entire $Q$ range.

In summary, we have reported a systematic SANS study of SWNTs suspensions. For the first time, we have unambiguously shown that isolated SWNTs can behave as rigid rods in suspension depending on the choice of surfactant. For these well dispersed suspensions, the scattering intensity follows a $Q^{-1}$ law over a $Q$ range of roughly one decade whose lower bound is consistent with independent measurements of tube length. The data also suggest that the rigid rod nanotubes can also form a loose 3-D network in suspension. Conversely, the scattered intensity from nanotube agglomerates in more aggregated suspensions follows a power law dependence with exponents in the range of -2 to -3 over the entire range of measured $Q$, suggesting some sort of network of ropes.

We gratefully acknowledge useful conversations with Tom Lubensky, Randy Kamien, Paul Janmey and Klus Kroy. This work was supported by ONR Grant N0001401-1-0657 and by the National Science Foundation MRSEC program Grant DMR0203378. AGY also acknowledges partial support from the NSF Grant DMR 00-79909 and NASA Grant NAG8-2172. We acknowledge the support of the National Institute of Standards and Technology, U.S. Department of Commerce, in providing facilities used in this work. Certain commercial materials and equipment are identified in this paper in order to specify adequately the experiment procedure. In no case does such identification imply recommendation by the National Institute of Standards and Technology nor does it imply that the material or equipment identified is necessarily the best available for this purpose. 


\section{References:}

[1] R. Saito, G. Dresselhaus and M. S. Dresselhaus, Physical Properties of Carbon Nanotubes, Imperial College Press: London, 1998.

[2] D. I. Svergun and L. A. Feigin, Structure analysis by small-angle x-ray and neutron scattering, Editor G. W. Taylor, Plenum Press, New York, 1987.

[3] D. W. Schaefer, J. Zhao, J. M. Brown, D. P. Anderson and D. W. Tomlin, Chem. Phys. Lett., 375 (2003) 369.

[4] R. Krishnamoorti, C. A. Mitchell and K. Yurekli, Macromoleucles (submitted).

[5] Y. W. Yip and B. I. Yakobson, International Conference on the Science and Application of Nanotubes, Boston College, July 6-11, 2002.

[6] M. M. J. Treacy, T. W. Ebbesen and J. M. Gibson, Nature 381 (1996) 678.

[7] E. M. Lifshitz and L. V. Landau, Theory of Elasticity, Butterworth-Heinemann: Oxford, 1980.

[8] W. Zhou, Y. H. Ooi, R. Russo, P. Papanek, D. E. Luzzi, J. E. Fischer, M. J. Bronikowski, P. A. Willis and R. E. Smalley, Chem. Phys. Lett., 350 (2001) 6.

[9] M. J. Bronikowski, P.A. Willis, D. T. Colbert, K. A. Smith and R.E. Smalley, J. Vac. Sci. Technol. A. 19 (2001) 1800.

[10] A. G. Rinzler, J. Liu, P. Nikolaev, C. B. Huffman, F. J. Rodriguez-Macias, P. J. Boul, A. H. Lu, D. Heymann, D. T. Colbert, R. S.Lee, J. E. Fischer, A. M. Rao, P. C. Eklund and R. E. Smalley, Applied Physics A 67 (1998) 29.

[11] M. F. Islam, E. Rojas, D. M. Bergey, A. T. Johnson and A. G. Yodh, Nano Lett., 3 (2) 269 (2003).

[12] F. Du, J. E. Fischer and K. I Winey, J. Polymer Sci. B: Polymer Physics (in press). 
[13] V. B. Fainerman, D. Möbius and R. Miller, Surfactants: chemistry, interfacial properties, applications, New York: Elsevier, 2001.

[14] P-G. de Gennes, Scaling Concepts in Polymer Physics, Cornell University Press: Ithaca, 1979. 


\section{Figure Captions}

Figure 1. (a) SANS intensity profiles for NaDDBS in $\mathrm{D}_{2} \mathrm{O}$ (no tubes). Note the broad correlation peak from spherical micelles at around $Q=0.07 \AA^{-1}$ for $c_{\mathrm{NaDDBS}}=0.5,1$ and $5 \mathrm{wt} \%$ but not at the lowest $c_{\mathrm{NaDDBS}}=0.05 \mathrm{wt} \%$. Also note the dramatic increase in neutron intensity with decreasing $Q$, starting between $0.003-0.005 \AA^{-1}$ in all samples. $(b, c)$ SANS intensity profiles for HiPco-NaDDBS and Tubes@Rice-NaDDBS in $\mathrm{D}_{2} \mathrm{O}$ respectively, with $c_{\mathrm{SWNTs}}=0.01,0.05$ and $0.1 \mathrm{wt} \%$. Nanotube:surfactant ratio was 1:10 for all SWNTs-NaDDBS suspensions. Both $0.1 \mathrm{wt} \%$ and $0.05 \mathrm{wt} \%$ samples exhibit $Q^{-1}$ behavior over a limited $\mathrm{Q}$ range; this is not detected in the most dilute sample. Excess surfactant contribution is also clearly evident from the correlation peak and the diverging intensity as $Q \rightarrow 0$. (d) Scattered intensity from solutions with fixed concentration of NaDDBS (1 wt \%), but with differing SWNT concentrations of $0,0.01,0.05$ and $0.1 \mathrm{wt} \%$. Our hypothesis was that as surfactant molecules adsorb onto the added SWNTs, the number of micelles will decrease and the intensity of the micelle correlation peak at $Q=$ $0.07 \AA^{-1}$ will also decrease. The micelle correlation peak at $Q=0.07 \AA^{-1}$ was only weakly affected by the addition of SWNTs, indicating that the majority of surfactant forms micelles. We also found that the SWNTs-NaDDBS complexes dominate $I$ for $Q<$ $0.07 \AA^{-1}$.

Figure 2. Subtraction of surfactant contribution from total $I(Q)$, for both HiPco and Tubes@Rice with 0.1 wt $\%$ and 0.05 wt $\%$ SWNT concentrations. Dashed and dotted lines correspond to the extreme assumptions of no excess surfactant and no surfactant on

the tubes. A $Q^{-1}$ slope is clearly visible over a $Q$ range from 0.003 to $0.02 \AA^{-1}$, no matter 
what fraction of surfactant $I(Q)$ is subtracted. The scattered intensities also exhibit a crossover from $Q^{-1}$ to $Q^{-2}$ at $\sim 0.004 \AA^{-1}$, suggesting the SWNTs form a dilute network with a mesh size of $\sim 160 \mathrm{~nm}$.

Figure 3. SANS scattering profiles from a suspension for which the contrast between surfactant and solvent has been matched by mixing $\mathrm{D}_{2} \mathrm{O}$ with $\mathrm{H}_{2} \mathrm{O}$ in the ratio 1:4.394 by weight. The concentration of HiPco is $0.02 \mathrm{wt} \%$ and the ratio of nanotube-to-surfactant is 1:10. After subtracting the incoherent background, the -2 slope at low $Q$ is quite apparent and a fairly convincing -1 slope above $Q=0.003 \AA^{-1}$ is observed.

Figure 4. $I(Q)$ for $0.1 \mathrm{wt} \%$ HiPco in $\mathrm{D}_{2} \mathrm{O}$ with $1 \mathrm{wt} \%$ Triton $\mathrm{X}-100$ (squares) and $1 \mathrm{wt} \%$ of Triton $\mathrm{X}-100$ in $\mathrm{D}_{2} \mathrm{O}$ (triangles). If we take $83 \%$ of the Triton $\mathrm{X}-100$ as the fraction remaining in the water phase to optimally remove the contribution of surfactant at high $Q$, then the resultant intensity profile (denoted by circles) exhibits a $Q^{-2}$ slope over the entire $Q$ range measured. 


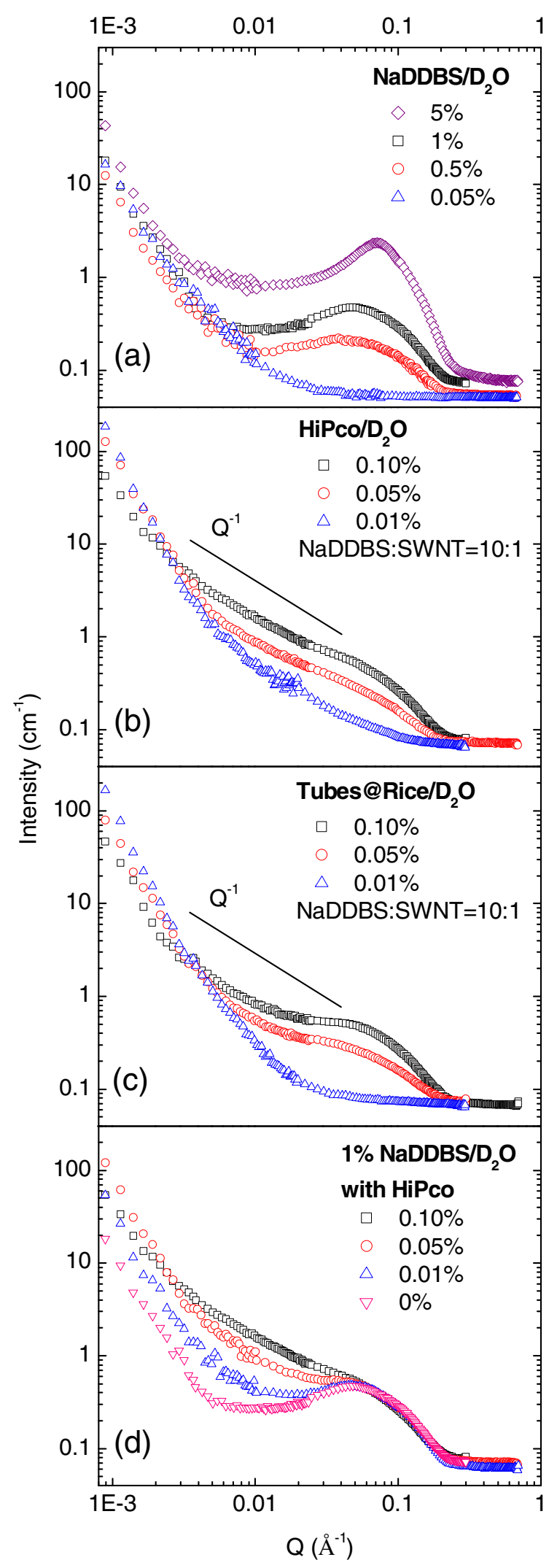

Fig.1 


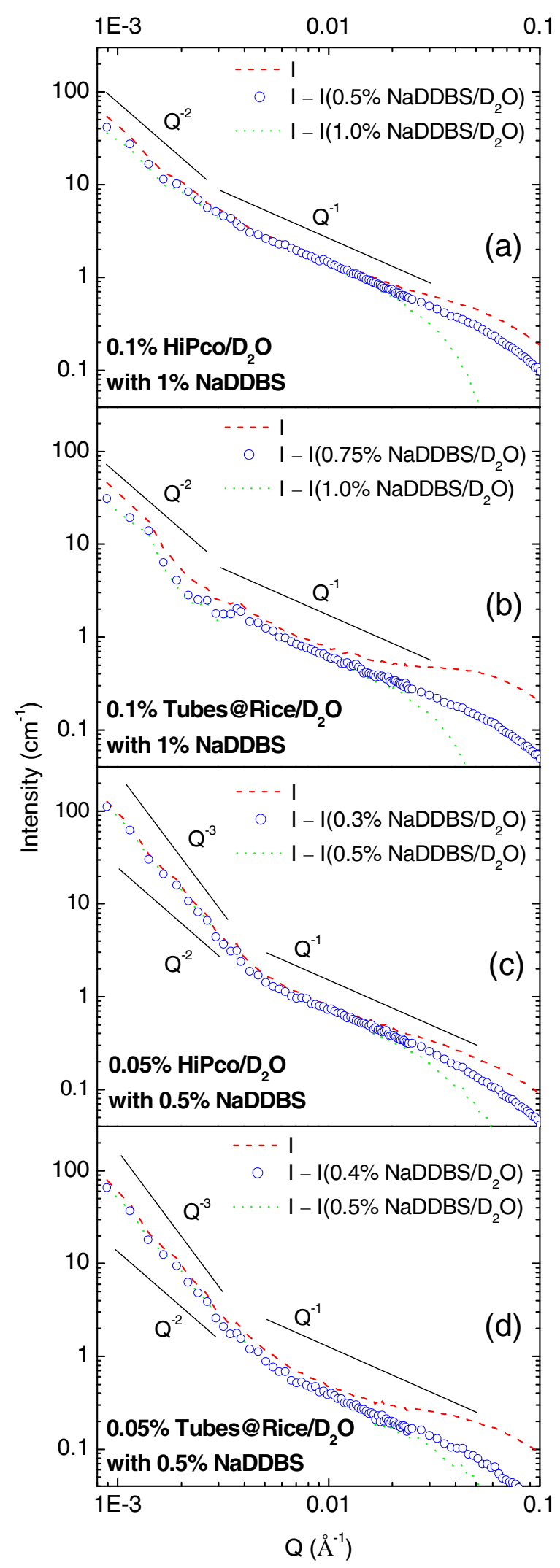

Fig.2 


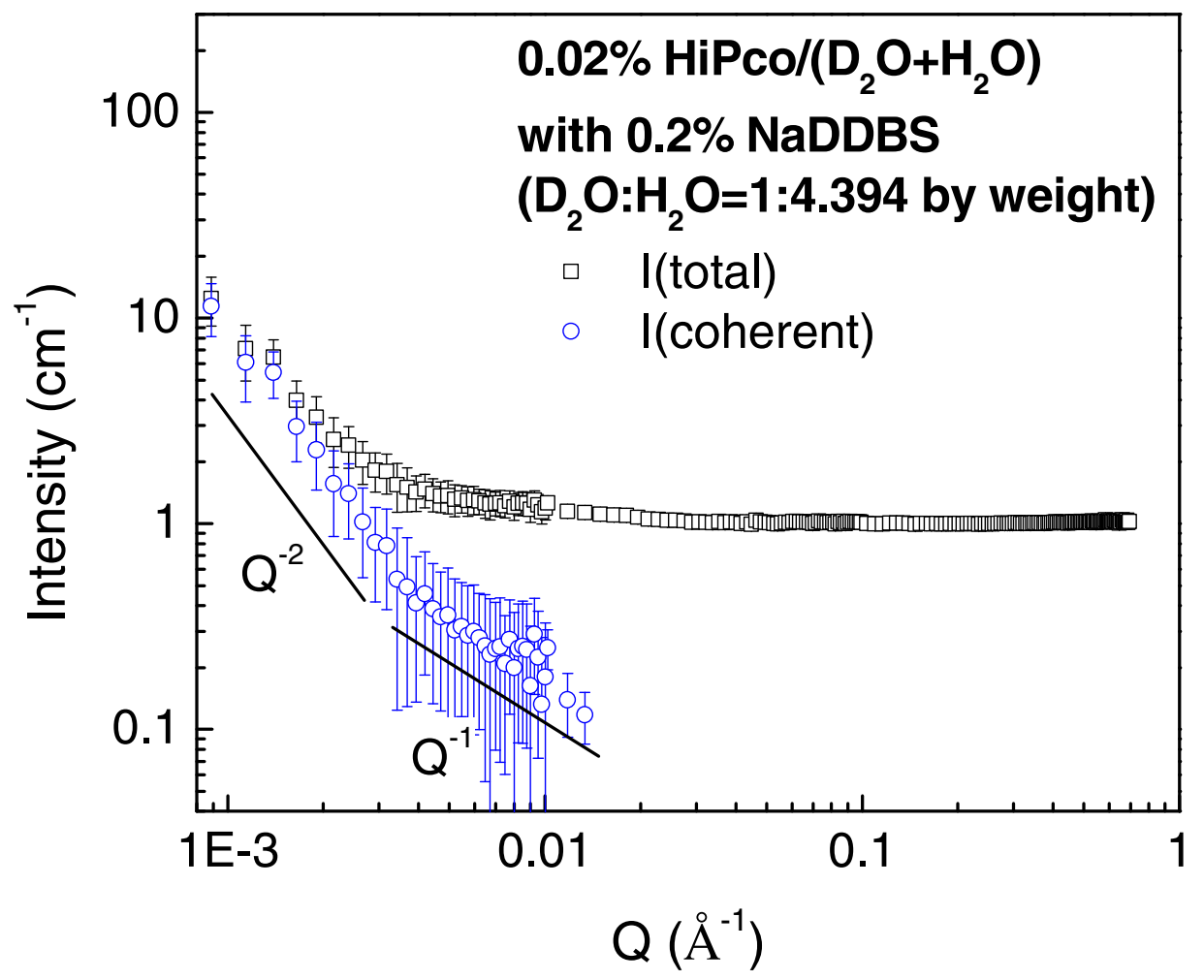

Fig.3 


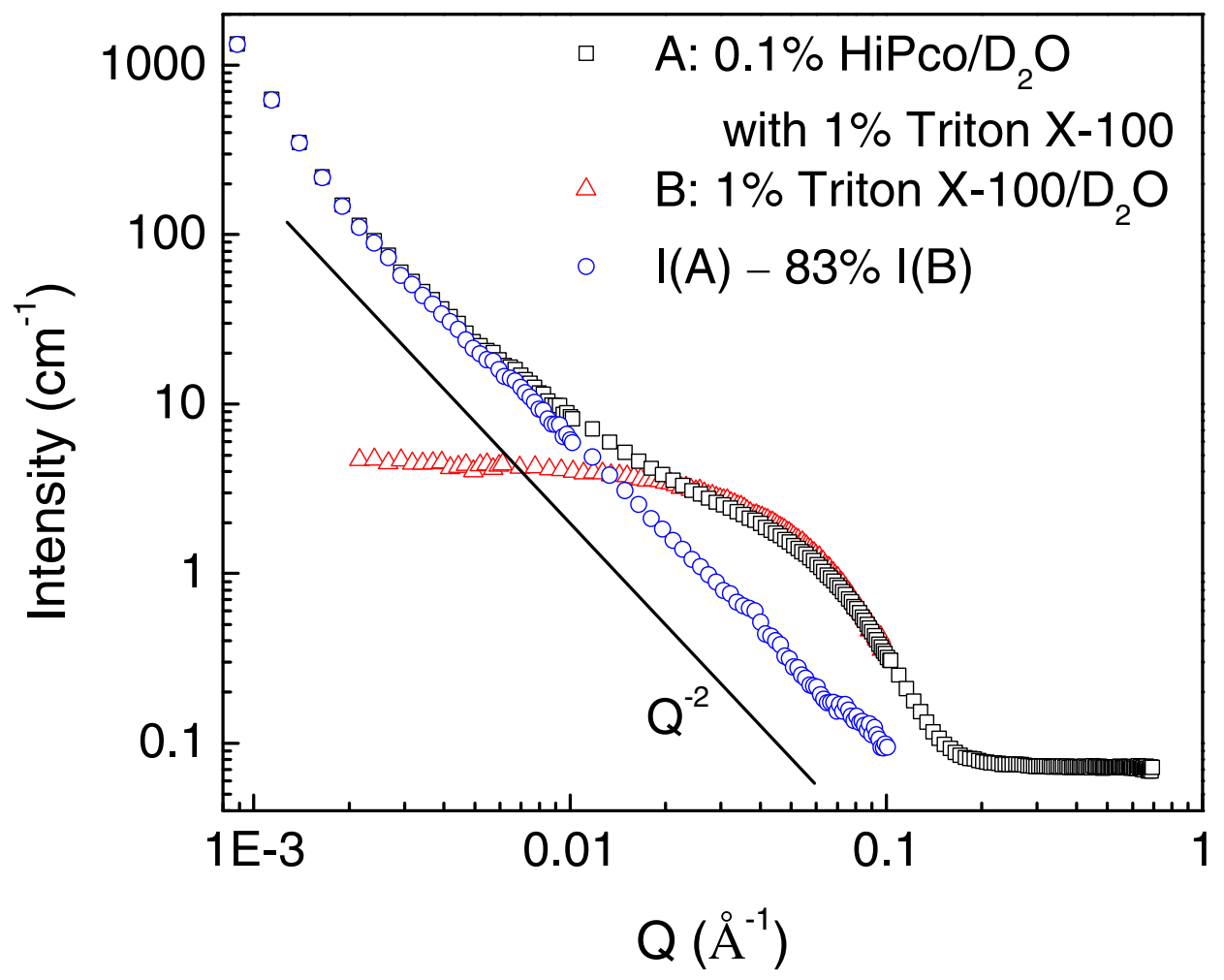

Fig.4 